\title{
Complications of Systemic Lupus Erythematosus in the Emergency Department
}

Joanna L. Marco, MD; Christine L. Chhakchhuak, MD

\section{Understanding and differentiating common from emergent complications of systemic lupus erythematous assists the clinician in recognizing and making appropriate treatment decisions in this complex patient population.}
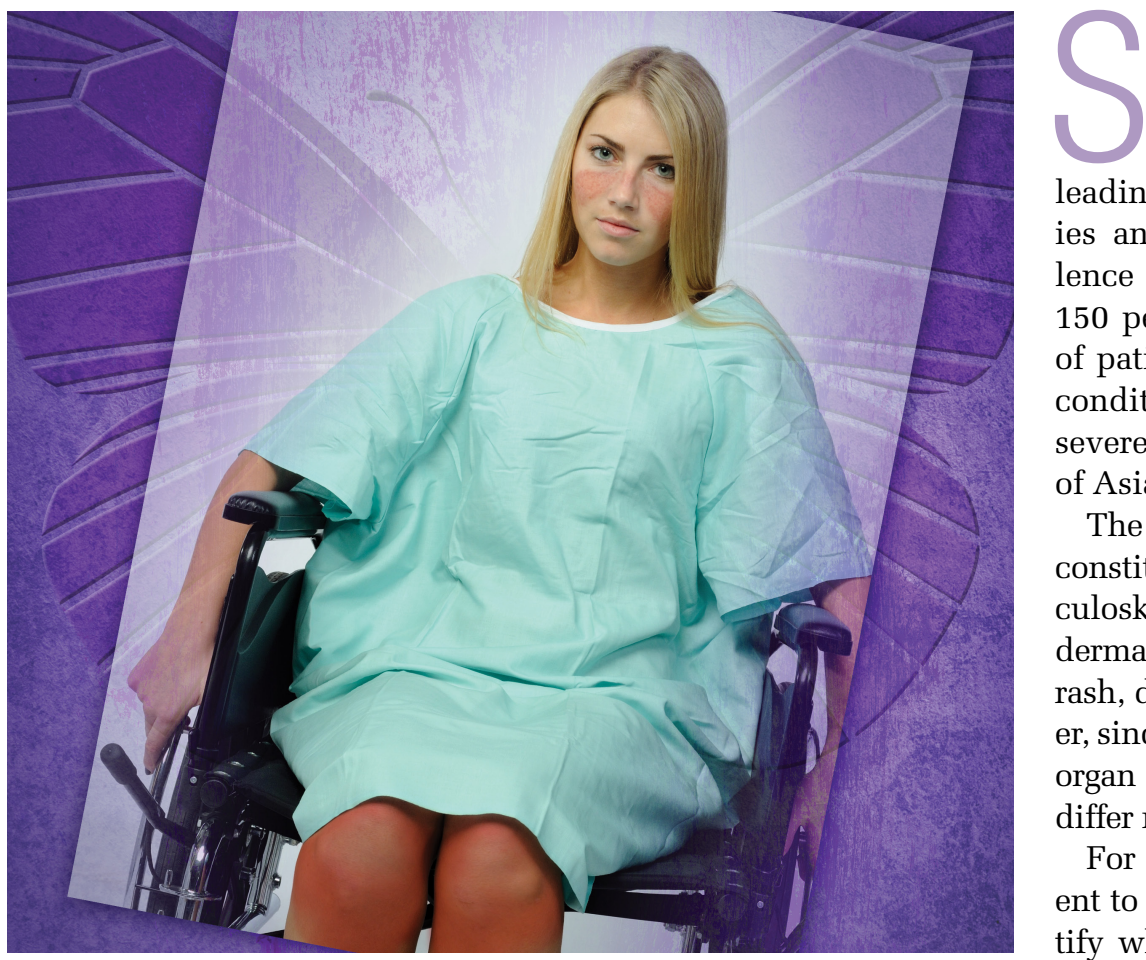

ystemic lupus erythematosus (SLE) is a chronic autoimmune disease characterized by the chronic activation of the immune system, leading to the formation of autoantibodies and multi-organ damage. The prevalence of SLE in the United States is 20 to 150 per 100,000 persons. ${ }^{1}$ Ninety percent of patients with SLE are women, and the condition is more common and often more severe among patients of black African or of Asian descent.

The most common symptoms of SLE are constitutional (fever or weight loss), musculoskeletal (arthralgias or myalgias), and dermatological (malar rash, photosensitive rash, discoid lupus, or oral ulcers). However, since SLE is known to affect nearly every organ system, clinical manifestations may differ markedly among patients (Table).

For patients with known SLE who present to the ED, it can be a challenge to identify whether their symptoms are due to a

Dr Marco is a resident, department of internal medicine, University of Cincinnati, Ohio. Dr Chhakchhuak is an assistant professor of medicine, department of internal medicine, division of immunology, allergy, and rheumatology, University of Cincinnati, Ohio.

Authors' Disclosure Statement: The authors report no actual or potential conflict of interest in relation to this article.

DOI: 10.12788/emed.2018.0075 
Table. Systemic Lupus Erythematous-Associated Conditions by Chief Complaint ${ }^{\mathrm{a}}$

\begin{tabular}{|c|c|c|c|}
\hline $\begin{array}{l}\text { Chief } \\
\text { Complaint }\end{array}$ & $\begin{array}{l}\text { SLE-Associated Conditions } \\
\text { (Emergent conditions bolded) }\end{array}$ & Suggested Workup & Clinical Pearls \\
\hline \multirow[t]{7}{*}{ Fever } & Active SLE & Chest X-ray & \multirow{7}{*}{$\begin{array}{l}\text { Double-stranded DNA and } \\
\text { C3 and C4 complements } \\
\text { can suggest general SLE } \\
\text { flare, but results may not } \\
\text { be available during ED visit. }\end{array}$} \\
\hline & Pericarditis & Urinalysis & \\
\hline & \multirow{2}{*}{$\begin{array}{l}\text { Infection (consider opportunistic infections } \\
\text { if immunocompromised) }\end{array}$} & Consider blood cultures & \\
\hline & & \multirow{2}{*}{$\begin{array}{l}\text { Other infectious workup based } \\
\text { on symptoms }\end{array}$} & \\
\hline & Arterial or venous thromboembolism & & \\
\hline & & \multirow{2}{*}{$\begin{array}{l}\text { Imaging if concern for } \\
\text { thromboembolic disease }\end{array}$} & \\
\hline & Diffuse alveolar hemorrhage & & \\
\hline \multirow[t]{4}{*}{ Joint pain } & Active SLE & Plain radiography & \multirow{3}{*}{$\begin{array}{l}\text { A single inflamed, painful } \\
\text { joint is less likely to be } \\
\text { caused by active SLE } \\
\text { and should prompt } \\
\text { consideration of septic } \\
\text { arthritis. }\end{array}$} \\
\hline & $\begin{array}{l}\text { Avascular necrosis (especially when hips or } \\
\text { knees are involved) }\end{array}$ & $\begin{array}{l}\text { Non-urgent MRI if avascular } \\
\text { necrosis is suspected }\end{array}$ & \\
\hline & \multirow[t]{2}{*}{ Septic arthritis } & \multirow[t]{2}{*}{$\begin{array}{l}\text { Arthrocentesis if septic arthritis } \\
\text { is suspected }\end{array}$} & \\
\hline & & & $\begin{array}{l}\text { Cover for Salmonella } \\
\text { and typical gram-positive } \\
\text { organisms with ceftriaxone } \\
\text { and vancomycin. }\end{array}$ \\
\hline \multirow[t]{4}{*}{ Rash } & Malar rash & \multirow{4}{*}{$\begin{array}{l}\text { Evaluate for systemic vasculitis } \\
\text { if cutaneous vasculitis is } \\
\text { present }\end{array}$} & \\
\hline & Photosensitive rash & & \\
\hline & Discoid lupus & & \\
\hline & Cutaneous vasculitis & & \\
\hline \multirow[t]{4}{*}{ Headache } & Primary headache disorder & \multirow{4}{*}{$\begin{array}{l}\text { Consider imaging or LP if } \\
\text { headache is new or different } \\
\text { from previous headaches, or } \\
\text { if there are other concerning } \\
\text { signs or symptoms }\end{array}$} & \multirow{4}{*}{$\begin{array}{l}\text { CT is not sensitive for } \\
\text { dural sinus thrombosis. } \\
\text { Choose MRI when this is } \\
\text { suspected. }\end{array}$} \\
\hline & CNS infection & & \\
\hline & Dural sinus thrombosis & & \\
\hline & CNS vasculitis & & \\
\hline \multirow{5}{*}{$\begin{array}{l}\text { Altered } \\
\text { mental } \\
\text { status }\end{array}$} & Lupus psychosis & \multirow{2}{*}{$\begin{array}{l}\text { Chest X-ray, urinalysis, and } \\
\text { blood cultures }\end{array}$} & \\
\hline & Stroke & & \\
\hline & Sepsis & Consider LP & \\
\hline & CNS infection & \multirow[t]{2}{*}{ Consider head CT or MRI } & \\
\hline & CNS vasculitis & & \\
\hline
\end{tabular}


Table. Systemic Lupus Erythematous-Associated Conditions by Chief Complainta (continued)

\begin{tabular}{|c|c|c|}
\hline $\begin{array}{l}\text { Chief } \\
\text { Complaint }\end{array}$ & $\begin{array}{l}\text { SLE-Associated Conditions } \\
\text { (Emergent conditions bolded) }\end{array}$ & Suggested Workup \\
\hline \multirow[t]{4}{*}{ Seizure } & Primary seizure disorder & $\begin{array}{l}\text { CT or MRI if no known history } \\
\text { of seizure disorder }\end{array}$ \\
\hline & Stroke & \multirow{3}{*}{ LP if meningitis is suspected } \\
\hline & CNS infection & \\
\hline & CNS vasculitis & \\
\hline \multirow{6}{*}{$\begin{array}{l}\text { Focal } \\
\text { neurological } \\
\text { deficit }\end{array}$} & SLE mononeuritis & \multirow{6}{*}{$\begin{array}{l}\text { CT or MRI if symptoms are } \\
\text { acute } \\
\text { LP if meningitis is suspected }\end{array}$} \\
\hline & Stroke & \\
\hline & CNS infection (including epidural abscess) & \\
\hline & CNS vasculitis & \\
\hline & Dural sinus thrombosis & \\
\hline & Transverse myelitis & \\
\hline \multirow{11}{*}{$\begin{array}{l}\text { Dyspnea or } \\
\text { cough }\end{array}$} & Pleuritis & Chest X-ray \\
\hline & Interstitial lung disease & Echocardiogram \\
\hline & Pulmonary hypertension & Complete blood count \\
\hline & Shrinking lung syndrome & Consider chest CT \\
\hline & Pulmonary embolism & \multirow[t]{7}{*}{ Consider cardiac biomarkers } \\
\hline & $\begin{array}{l}\text { Pulmonary infection, including } \\
\text { opportunistic infection }\end{array}$ & \\
\hline & Acute lupus pneumonitis & \\
\hline & Diffuse alveolar hemorrhage & \\
\hline & $\begin{array}{l}\text { Libman-Sacks endocarditis with valvular } \\
\text { dysfunction }\end{array}$ & \\
\hline & Acute coronary syndrome & \\
\hline & Autoimmune hemolytic anemia & \\
\hline
\end{tabular}

minor lupus flare that can be managed as an outpatient, a presentation of urgent or emergent conditions caused by SLE, or a condition unrelated to lupus. This article reviews the most common and emergent complications of SLE by organ system to assist emergency physicians (EPs) in better diagnosing and managing this complicated disease.
General Acute-Care Management While a patient's presentation could be secondary to a lupus-related complication, consideration must always be given to common conditions that are not related to SLE. Biomarkers such as erythrocyte sedimentation rate, C-reactive protein, C3 and C4 complement, and double-stranded 
Table. Systemic Lupus Erythematous-Associated Conditions by Chief Complainta (continued)

\begin{tabular}{|c|c|c|c|}
\hline $\begin{array}{l}\text { Chief } \\
\text { Complaint }\end{array}$ & $\begin{array}{l}\text { SLE-Associated Conditions } \\
\text { (Emergent conditions bolded) }\end{array}$ & Suggested Workup & Clinical Pearls \\
\hline \multirow[t]{11}{*}{ Chest pain } & Pleuritis & Chest X-ray & \multirow{11}{*}{$\begin{array}{l}\text { Patients with SLE } \\
\text { have a } 10 \text {-fold higher } \\
\text { risk of coronary artery } \\
\text { disease than the general } \\
\text { population, so acute } \\
\text { coronary syndrome should } \\
\text { be considered even in } \\
\text { young patients without } \\
\text { other risk factors. }\end{array}$} \\
\hline & Pericarditis & Echocardiogram & \\
\hline & Interstitial lung disease & Cardiac biomarkers & \\
\hline & Pulmonary hypertension & Consider chest CT & \\
\hline & Shrinking lung syndrome & \multirow{7}{*}{$\begin{array}{l}\text { Consider cardiac stress } \\
\text { testing }\end{array}$} & \\
\hline & Pulmonary embolism & & \\
\hline & $\begin{array}{l}\text { Pulmonary infection, including } \\
\text { opportunistic infection }\end{array}$ & & \\
\hline & Acute lupus pneumonitis & & \\
\hline & Diffuse alveolar hemorrhage & & \\
\hline & $\begin{array}{l}\text { Libman-Sacks endocarditis with valvular } \\
\text { dysfunction }\end{array}$ & & \\
\hline & Acute coronary syndrome & & \\
\hline \multirow{4}{*}{$\begin{array}{l}\text { Abdominal } \\
\text { pain }\end{array}$} & Pancreatitis & Liver function tests & \\
\hline & Peptic ulcer disease & Lipase & \\
\hline & Mesenteric vasculitis & Lactate & \\
\hline & & $\begin{array}{l}\text { Abdominal CT if severe pain or } \\
\text { no other etiology found }\end{array}$ & \\
\hline
\end{tabular}

aln addition to SLE-associated conditions presented here, conditions that are common in the general population should always be considered. Abbreviations: CNS, central nervous system; CT, computed tomography; LP, lumbar puncture; MRI, magnetic resonance imaging; SLE, systemic lupus erythematous.

DNA levels can be helpful in assessing lupus disease activity and differentiating a lupus-related complication from an unrelated event. Comparing these biomarkers to the patient's baseline values can be informative; however, depending on the laboratory facilities, test results may not be available during an ED visit. Lastly, infections should be considered more strongly than usual in the differential diagnosis due to the immunocompromised status of a substantial proportion of these patients, by virtue of their disease or the cytotoxic medications used for treatment.

\section{Musculoskeletal Complications}

\section{Common Complications}

Polyarthralgias and Polymyalgias. More than $90 \%$ of SLE patients experience polyarthralgias and polymyalgias. Physical examination findings may be normal, even when joint pain is present, which is often due to mild synovitis. In some cases, Jaccoud arthropathy is seen, which presents as deformities such as swan neck deformities and ulnar deviations that are characteristically reducible on manipulation (Figures 1a and 1b). These deformities are not caused by direct joint damage, but by 

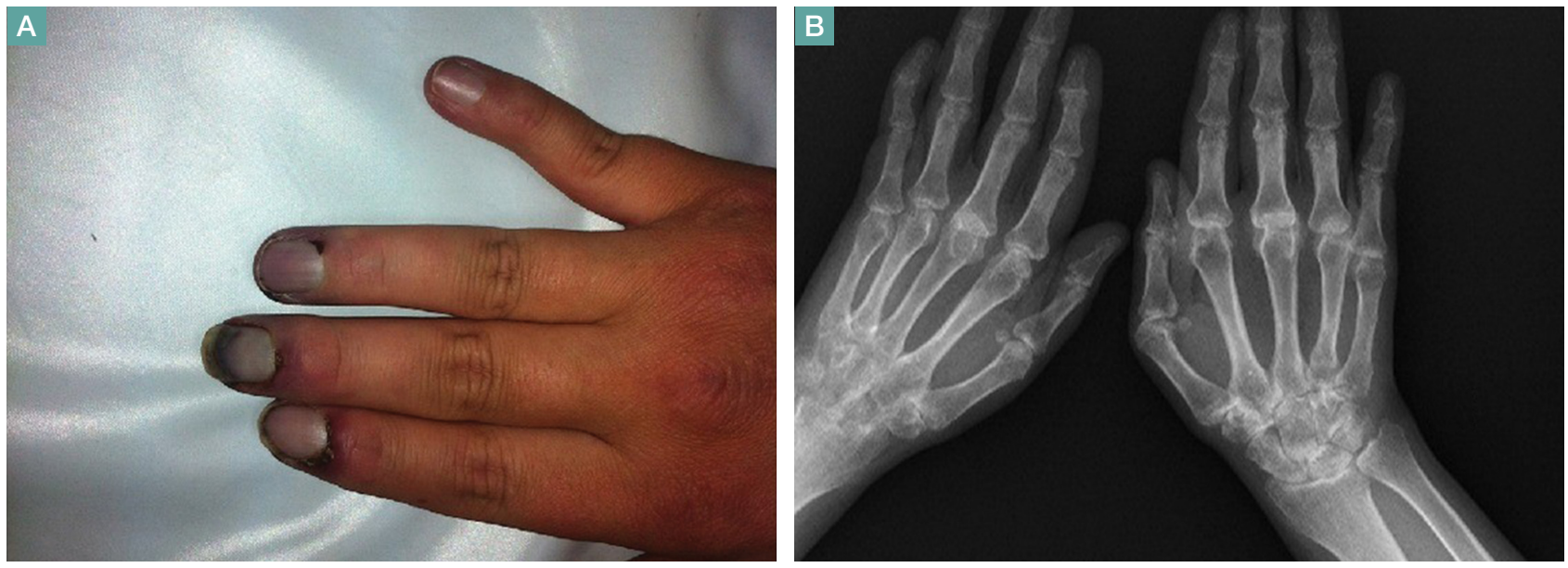

Figure 1. (A) Photo demonstrating swan neck deformities of the second, third, and fifth digits of the right hand. (B) Normal bilateral hand X-ray suggesting Jaccoud arthropathy.

Reproduced with permission from Küçükşahin, et al. ${ }^{28}$

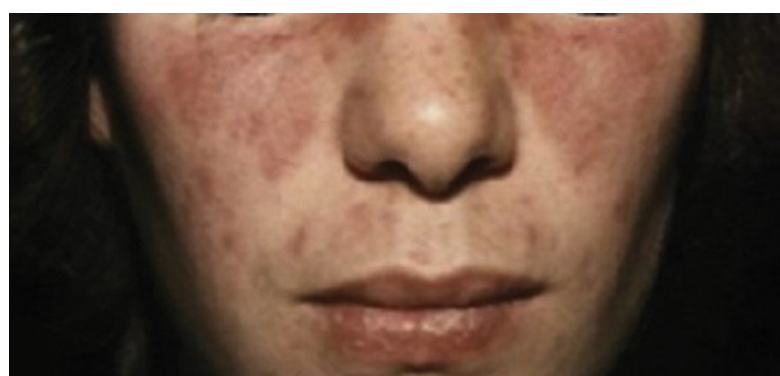

Figure 2. Photo demonstrating a malar rash with sparing of the nasolabial fold in a patient with systemic lupus erythematous.

Reproduced with permission from Uva et al. ${ }^{29}$

chronic tenosynovitis and the resulting laxity of tendons and ligaments. ${ }^{1}$ Classically, plain radiographic imaging reveals nonerosive joint changes. Muscle and joint pains may worsen with disease progression or flare.

Avascular Necrosis. Avascular necrosis affects $5 \%$ to $12 \%$ of SLE patients. ${ }^{2}$ Most commonly, this involves the femoral head, but it may also involve the femoral condyle or tibial plateau. Patients may present with acute or subacute onset of pain in the groin or buttocks when the femoral head is involved, or in the knee when the femoral condyle or tibial plateau is involved. Plain radiographs may reveal joint-space narrowing and other evidence of degenerative joint disease. Magnetic resonance imaging (MRI) is more sensitive in diagnosing avascular necrosis, and may be indicated when clinical suspicion is high despite negative plain radiographs, although this would not typically need to be performed urgently in the ED. ${ }^{2}$ While analgesics and physical therapy may provide some pain relief to patients with avascular necrosis, this condition generally requires nonemergent operative intervention.

\section{Emergent Complications}

Septic Arthritis. When a patient with SLE presents with an isolated swollen joint, septic arthritis should be suspected, and diagnosis should be confirmed by arthrocentesis. Synovial fluid samples showing a white blood cell count greater than $50 \times 10^{9} / \mathrm{L}$ suggest infection, which can be confirmed by gram stain and cultures.

For reasons that remain unclear, but may involve primary immune defects and the use of immunosuppressant medications, patients with SLE are predisposed to Salmonella joint infections. In one study, 59\% of septic arthritis cases in patients with SLE were due to Salmonella species; therefore, treatment for septic arthritis in this population should include ceftriaxone in 
addition to vancomycin for typical organisms, such as Staphylococcus and Streptococcus species. ${ }^{3}$

\section{Cutaneous Manifestations}

\section{Common Complications}

Malar Rash. Eighty percent to $90 \%$ of patients with SLE have dermatological involvement, ${ }^{1}$ the most common finding of which is the malar or butterfly facial rash, which appears as raised erythema over the bridge of the nose and cheeks while sparing the nasolabial folds (Figure 2).

Discoid Lupus. Chronic discoid lupus appears as a scarring rash often found on the face, ears, and scalp. These patients may also exhibit a photosensitive rash, which consists of an erythematous eruption if acute, or annular scaly lesions if subacute.

Oral and Nasal Ulcerations. Common mucous membrane findings include oral or nasal ulcers, which are typically painless.

Worsening of any of these skin findings may be associated with disease flare. Secondary bacterial infection of lupus rashes or ulcerations is uncommon, although cellulitis should be considered when a rash is unilateral, not in a sun-exposed area, or is otherwise different from the patient's typical lupus rash. Sun avoidance and topical corticosteroids are the mainstays of treatment of dermatological disease in SLE.

\section{Emergent Complications}

Systemic Vasculitis. Patients with SLE are susceptible to vasculitis. Although isolated cutaneous vasculitis is not typically an emergent condition, it may portend systemic vasculitis. Any palpable purpura or other evidence of cutaneous vasculitis should prompt a careful review of systems and basic laboratory workup for systemic vasculitis, which can involve the kidneys, lungs, central or peripheral nervous system, or gastrointestinal tract.

Symptoms of systemic vasculitis may include fevers, chills, chest pain, cough, hemoptysis, abdominal pain, and changes in color or amount of urine. Laboratory workup should be tailored to symptoms, and may include basic metabolic panel, liver function tests, complete blood count, and urinalysis. ${ }^{4}$

Digital Gangrene. Patients with SLE may also develop digital gangrene related to severe Raynaud phenomenon, vasculitis, or thromboembolism. Pharmacological treatment with vasodilators such as sildenafil, endothelin receptor antagonists, or intravenous prostacyclins may be needed. ${ }^{5}$ To save the involved digit, vascular surgery services should be consulted urgently. ${ }^{6}$

\section{Renal Complications}

\section{Common Complications}

Chronic Kidney Disease. Chronic kidney disease (CKD) is common among SLE patients, especially among those with a history of lupus nephritis. ${ }^{7}$ Patients with CKD may have persistently elevated serum creatinine, chronic hypertension, and/or chronic peripheral edema. Patients presenting with new development of hypertension, peripheral edema, hematuria, or polyuria should be screened for lupus nephritis with urinalysis and serum creatinine. Elevated creatinine or new or worsening proteinuria or hematuria should prompt consultation with nephrology services.

\section{Emergent Complications}

Lupus Nephritis. About $50 \%$ of SLE patients will develop lupus nephritis during the course of their lives, ${ }^{1}$ which may present as nephrotic disease with significant proteinuria, peripheral edema, and low serum albumin, or as nephritic disease, with increased serum creatinine and hematuria. Acute kidney injury in SLE patients should generally prompt admission for workup of reversible causes and evaluation for lupus nephritis, which often includes renal biopsy. ${ }^{8}$

\section{Neuropsychiatric Complications Common Complications}

Neuropsychiatric lupus is a broad category that includes 19 manifestations of SLE in ...due to the

variety of

neurological

emergencies...

the threshold

to obtain

imaging on

SLE patients

with any new

neurological

complaints

should be low. 
Approximately half of SLE patients who present to the ED with acute abdominal pain are found to have either mesenteric vasculitis or pancreatitis... the central and peripheral nervous systems. ${ }^{9}$ Conditions range from depression or chronic headaches to seizures or psychosis.

Mood and Anxiety Disorders. Anxiety and depression have been observed in up to $75 \%$ of SLE patients. ${ }^{1}$ Mood and anxiety disorders are likely influenced by the psychosocial elements of this chronic disease, as well as by direct effects of SLE on the brain. ${ }^{1}$

Peripheral Neuropathy. Approximately $10 \%$ of SLE patients have a peripheral neuropathy, which generally presents as a mononeuritis (either single or multiplex), rather than the stocking-glove distribution seen in other systemic causes of neuropathy. ${ }^{10}$

Headache. Headache disorders may also develop in SLE patients, and tend to have similar patterns to primary headache disorders in the general population. In most cases, treatment for headache in SLE patients is similar to that of the general population. ${ }^{11}$ However, if a patient presents with concerning findings, such as focal neurological deficit, meningismus, or fever, or if the headache is new-onset or different from previous headaches, further investigation should be considered, including a head computed tomography (CT) scan and lumbar puncture (LP).

\section{Emergent Complications}

In general, due to the variety of neurological emergencies that may present with SLE, and the subtlety with which true emergencies may present in this population, the threshold to obtain imaging on SLE patients with any new neurological complaints should be low.

Cerebrovascular Accidents. Patients with SLE are susceptible to cerebrovascular accidents (CVAs), typically from occlusive or embolic causes. Etiologies may include primary central nervous system (CNS) vasculitis, embolic disease from antiphospholipid syndrome (APS), or embolic disease from a Libman-Sacks endocarditis. ${ }^{12}$
Successful thrombolysis has been reported in SLE patients presenting with stroke, but it remains controversial due to risk of hemorrhagic conversion if CNS vasculitis, rather than embolism, is the cause. ${ }^{13}$ Proper imaging and consultation with a neurologist familiar with the disease is critical for early treatment decisions.

Seizures. Fifteen percent to $35 \%$ of SLE patients may develop seizures. These may be focal or generalized, but generalized tonic-clonic seizures tend to be more common in SLE patients. ${ }^{2}$ Workup and management of seizures in SLE patients is the same as in the general population.

Sinus Thrombosis. Dural sinus thrombosis often presents as a new-onset headache, sometimes with focal neurological deficits. The diagnosis of dural sinus thrombosis can be challenging, as CT imaging studies may be falsely negative. There should be a low threshold for obtaining MRI/magnetic resonance angiography (MRA) in SLE patients presenting with a new-onset headache. ${ }^{14}$

CNS Vasculitis. Patients with SLE are also susceptible to CNS vasculitis, which can manifest as seizures, psychosis, cognitive decline, altered mental status, or coma. Magnetic resonance imaging/MRA studies may suggest the diagnosis, but if this is equivocal, angiography or even brain biopsy may be needed to make the diagnosis. Unless the patient's symptoms are very mild (eg, mild cognitive decline), she or he should be admitted for diagnostic workup and consideration of aggressive immunosuppressive therapy. ${ }^{2}$

Transverse Myelitis and Spinal Artery Thrombosis. Acute loss of lower limb sensation or motor function in SLE patients may be caused by transverse myelitis or spinal artery thrombosis. Epidural abscess should also be considered, especially if the patient is immunocompromised. ${ }^{2}$

Infection. A CNS infection should be considered in any SLE patient presenting with new neurological complaints. Fever or meningismus, especially in conjunction with headache or focal neurological 
deficits, should prompt an LP and consideration for imaging. Immunocompromised patients are at increased risk for common organisms as well as atypical organisms, such as fungus or mycobacteria. ${ }^{15}$

\section{Pulmonary Complications}

\section{Common Complications}

Pleuritis. Many patients with SLE develop pleuritis, with or without effusion. This may be treated with nonsteroidal antiinflammatory drugs, or corticosteroids if symptoms are more severe. Pleuritis is the most common respiratory complication of SLE, but due to the number of serious cardiopulmonary complications associated with SLE, pleuritis should be a diagnosis of exclusion.

Interstitial Lung Disease. Interstitial lung disease may be caused by SLE or may be medication-induced. This commonly presents as subacute or chronic dyspnea and/ or cough. Patient workup may be done on an outpatient basis with high resolution chest CT and pulmonary function testing.

Pulmonary Hypertension. Patients with SLE may develop pulmonary hypertension, either directly due to SLE or from chronic thromboembolic disease. In general, pulmonary hypertension is managed as an outpatient, but may require emergent inpatient treatment if the condition is rapidly progressive or associated with right heart failure.

Shrinking Lung Syndrome. This condition may cause subacute or chronic dyspnea and pleuritic chest pain. Shrinking lung syndrome is caused by diaphragmatic dysfunction rather than from a primary disease of the lungs, and it is characterized by a restrictive pattern on pulmonary function testing and an elevated hemidiaphragm. Shrinking lung syndrome typically responds well to immunosuppressive therapy. ${ }^{16}$

\section{Emergent Conditions}

Pulmonary Embolism. A pulmonary embolism should be strongly considered in any patient with SLE presenting with the appropri

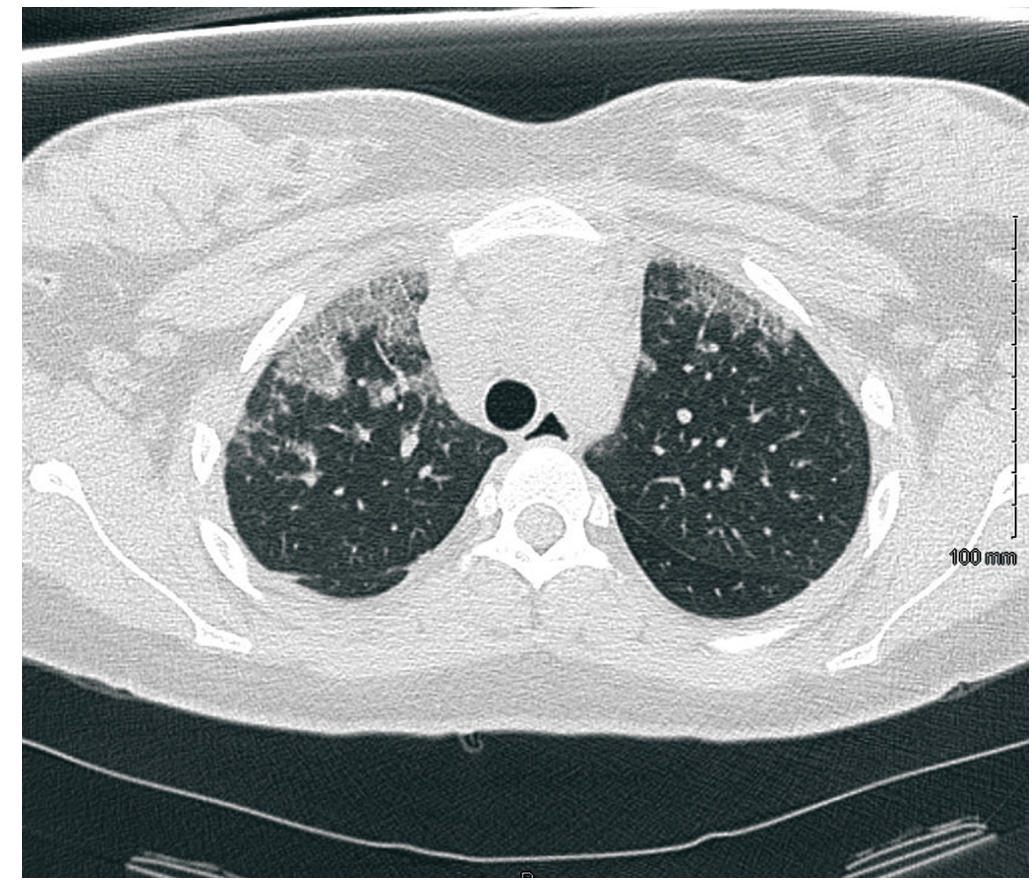

Figure 3. Computed tomography image of the chest revealing anterior ground-glass opacities in a patient with acute lupus pneumonitis.

Reproduced with permission from Nishant Gupta, MD.

ate clinical picture. Patients with APS are at particularly high risk for thromboembolic disease. However, even SLE patients without this APS are known to be at an increased risk of developing thromboembolism compared to the general public. ${ }^{17}$ Pulmonary embolism in SLE patients should be diagnosed and treated in the usual manner.

Pneumonia. Immunosuppressed patients are susceptible to opportunistic pulmonary infections as well as typical community pathogens. Fungal or mycobacterial infections may be suspected with a more subacute onset of symptoms.

Acute Lupus Pneumonitis. This serious condition may present with severe pneumonia-like signs and symptoms, including fever, cough, dyspnea, hypoxia, and infiltrates on chest radiograph (Figure 3).

Acute lupus pneumonitis is caused by disease flare, and not by infection, although it may not be possible to distinguish it from pneumonia in the ED setting. The mortality 
rate of acute lupus pneumonitis is as high as $50 \%$, and survivors often progress to chronic interstitial pneumonitis. ${ }^{1}$

Diffuse Alveolar Hemorrhage. A rare complication with a mortality rate of $50 \%$ to $90 \%$, SLE patients who develop diffuse alveolar hemorrhage may present with fever, cough, dyspnea, and hypoxia. ${ }^{18}$ The condition may be suggested by infiltrates on chest radiograph, a drop in hemoglobin representing bleeding into the lungs, and/or hemoptysis. However, the absence of hemoptysis does not rule out diffuse alveolar hemorrhage, so clinical suspicion should remain high, even in the absence of this symptom.

Because emergent pulmonary conditions often present with similar symptoms, most patients with acute or new-onset symptoms will require admission for diagnostic workup (likely to include chest CT scan and/or bronchoscopy with bronchoalveolar lavage), as well as for close monitoring and initiation of treatment. If hypoxia or respiratory distress is severe, or if diffuse alveolar hemorrhage is suspected, admission to the intensive care unit (ICU) should be considered. We suggest that antibiotics be started in the ED when pneumonia is part of the differential diagnosis. As in the general population, coverage should be chosen based on the patient's risk factors for antibiotic-resistant organisms. Initiation of corticosteroid therapy or other changes in immune therapy can be delayed until the EP consults with rheumatology and/or pulmonology services.

\section{Cardiac Complications}

\section{Common Complications}

Pericarditis. Pericarditis with or without pericardial effusion is very common in SLE patients and is usually related to lupus itself, rather than an infectious etiology. Patients may present with substernal, positional chest pain, tachycardia, and diffuse ST-segment elevation on electrocardiogram. Most effusions are small, asymptomatic, and discovered incidentally.
However, among patients with symptomatic pericardial effusions, tamponade can be present in $21 \% .{ }^{19}$ Corticosteroid therapy is often required to treat SLE-associated pericarditis, but colchicine is being explored as a possible steroid-sparing agent in this patient population..$^{20,21}$

Valvular Abnormalities. Approximately 60\% of SLE patients have valvular abnormalities detectable by echocardiography. The most common abnormalities in one study were valvular thickening or regurgitation. ${ }^{22}$ Many of these abnormalities occurred in asymptomatic patients and never progressed to clinical disease in a 5-year follow-up. However, patients with any valvular abnormality were more likely to develop complications, including stroke, peripheral embolism, infective endocarditis, need for valve replacement, congestive heart failure, or death. ${ }^{22}$

\section{Emergent Complications}

Acute Coronary Syndrome. Even in relatively young patients, acute coronary syndrome (ACS) should be considered in SLE patients presenting with chest pain, as this patient population has a 10 -fold higher risk of developing coronary artery disease (CAD) than the general population, and SLE patients with CAD often lack traditional risk factors, such as advanced age, family history, or metabolic syndrome. ${ }^{1}$

A high clinical suspicion should be maintained even in patients who would traditionally be considered low-risk. The EP should have a low-threshold for ECG, cardiac biomarker testing, and stress testing for SLE patients presenting with chest pain. The treatment of ACS in SLE patients is the same as in the general population.

Libman-Sacks Endocarditis. A sterile, fibrinous valvular vegetation, Libman-Sacks endocarditis is unique to patients with SLE. When present, patients usually develop a subacute or chronic onset of dyspnea or chest pain. However, patients may become acutely ill if they develop severe valvular regurgitation. Additionally, the valve dam- 
age from Libman-Sacks endocarditis can predispose patients to developing infective endocarditis. ${ }^{20}$

\section{Hematological Complications \\ Common Complications}

Patients with SLE commonly have mild-tomoderate leukopenia (especially lymphopenia), anemia, and thrombocytopenia. This may be related to the disease process or may be secondary to prescribed medications. A comparison to recent baseline laboratory studies should be sought if there is suspicion for new or worsening cytopenia.

Antiphospholipid Syndrome. Nearly $40 \%$ of SLE patients also have APS, which is defined by a clinical history of thrombosis in conjunction with one of the antiphospholipid antibodies (anticardiolipin, anti-beta-2-glycoprotein, lupus anticoagulant). Antiphospholipid syndrome causes both venous and arterial thrombosis and may be associated with recurrent miscarriage. Acute thrombotic events should be treated with heparin or enoxaparin and transitioned to warfarin. The new generation of direct oral anticoagulants have not been well studied in APS, though, multiple small case series suggest a higher thrombotic risk with these drugs than with warfarin. ${ }^{23}$ Patients who have recurrent venous thromboembolism, or who have any arterial thromboembolism should be on lifelong anticoagulation therapy. ${ }^{2}$

\section{Emergent Complications}

Thrombocytopenia. Severe thrombocytopenia or hemolytic anemia can be life-threatening, and often requires inpatient admission for immunosuppressive therapy, monitoring, and supportive care.

Catastrophic Antiphospholipid Syndrome. This condition should be suspected in patients with SLE who present with multiple sites of thrombosis or new multi-organ damage. Catastrophic APS (CAPS) may occur in SLE patients who have no prior history of APS. Since the mortality rate for CAPS approaches $50 \%$, these patients require anticoagulation, immunosuppres- sant therapy (high-dose corticosteroids, cyclophosphamide, and/or plasma exchange), and admission to the ICU. ${ }^{24}$

\section{Gastrointestinal Complications Common Complications}

Intestinal Pseudo-obstruction. Dysphagia related to esophageal dysmotility is present in up to $13 \%$ of SLE patients. ${ }^{25}$ Intestinal pseudo-obstruction may be seen in SLE patients, and is characterized by symptoms of intestinal obstruction caused by decreased intestinal motility, rather than from mechanical obstruction. Presenting symptoms may be acute or chronic, and include nausea, vomiting, and abdominal distension. Abdominal CT studies will show dilated bowel loops without evidence of mechanical obstruction. Manometry reveals widespread hypomotility. Intestinal pseudo-obstruction typically responds well to corticosteroids and other immunosuppressant therapies. ${ }^{26}$

\section{Emergent Conditions}

Acute Abdominal Pain. Approximately half of SLE patients who present to the ED with acute abdominal pain are found to have either mesenteric vasculitis or pancreatitis, both of which are thought to be related to SLE disease activity. ${ }^{27}$ Other causes of acute abdominal pain that are common in the general population remain common in SLE patients, including gallbladder disease, gastroenteritis, appendicitis, and peptic ulcer disease.

Mesenteric Vasculitis. Also known as lupus enteritis, mesenteric vasculitis is a unique cause of acute abdominal pain in SLE patients. The condition presents with acute, diffuse abdominal pain and may be associated with nausea and vomiting, diarrhea, or hematochezia. Abdominal CT findings suggestive of diffuse enteritis support the diagnosis. Medical management with pulse-dose corticosteroids and supportive care is generally sufficient, but if bowel necrosis or intestinal perforation is present or suspected, surgical consultation should be obtained immediately. ${ }^{15}$ 


\section{Conclusion}

Complications of SLE are diverse and may be difficult to diagnose. Understanding the common and emergent complications of SLE will help the EP to recognize severe illness and make appropriate treatment decisions in this complex patient population.

\section{References}

1. Dall'Era M, Wofsy D. Clinical Features of Systemic Lupus Erythematosus. In: Firestein GS et al, eds. Kelley and Firestein's Textbook of Rheumatology. 10th ed. Philadelphia, PA: Elsevier; 2017.

2. Dvorkina O, Ginzler EM. Clinical features of systemic lupus erythematosus. In: Hochberg MC, ed. Rheumatology. 6th ed. Philadelphia, PA: Elsevier; 2015.

3. Huang JL, Hung JJ, Wu KC, Lee WI, Chan CK, Ou LS. Septic arthritis in patients with systemic lupus erythematosus: salmonella and nonsalmonella infections compared. Semin Arthritis Rheumatol. 2006;36(1):61-67. doi:10.1016/j.semarthrit.2006.04.003

4. Barile-Fabris L, Hernández-Cabrera MF, BarraganGarfias JA. Vasculitis in systemic lupus erythematosus. Curr Rheumatol Rep. 2014;16(9):440. doi:10.1007/s11926-014-0440-9.

5. Campion EW, Wigley FM, Flavahan NA. Raynaud's phenomenon. N Engl J Med. 2016;375(6):556-565. doi:10.1056/NEJMra1507638.

6. Bouaziz JD, Barete S, Le Pelletier F, et al. Cutaneous lesions of the digits in systemic lupus erythematosus: 50 cases. Lupus. 2007;16(3):163-167.

7. Pokroy-Shapira E, Gelernter I, Molad Y. Evolution of chronic kidney disease in patients with systemic lupus erythematosus over a long-period follow-up: a single-center inception cohort study. Clin Rheumatol. 2014;33(5):649-657. doi:10.1007/s10067-014-2527-0.

8. Almaani S, Meara A, Rovin BH. Update on lupus nephritis. Clin J Am Soc Nephrol. 2017;12(5):825835. doi:10.2215/CJN.05780616.

9. The American College of Rheumatology nomenclature and case definitions for neuropsychiatric lupus syndromes. Arthritis Rheumatol. 1999;42(4):599-608.

10. Oomatia A, Fang H, Petri M, et al. Peripheral neuropathies in systemic lupus erythematosus: clinical features, disease associations, and immunologic characteristics evaluated over a twenty-five year study period. Arthritis Rheumatol. 2014;66(4):1000-1009.

11. Mitsikostas DD, Sfikakis PP, Goadsby PJ. A metaanalysis for headache in systemic lupus erythematosus: the evidence and the myth. Brain. 2004;127(pt 5):1200-1209.

12. Timlin H, Petri M. Transient ischemic attack and stroke in systemic lupus erythematosus. Lupus. 2013;22(12):1251-1258. doi:10.1177/0961203313497416.

13. Majdak MR, Vuletić V. Thrombolysis for acute stroke in patient with systemic lupus erythematosus: a case report. J Neurol Sci. 2016;(361):7-8. doi:10.1016/j. jns.2015.12.014.

14. Chen WL, Chang SH, Chen JH, Wu YL. Isolated headache as the sole manifestation of dural sinus thrombosis: a case report with literature review. Am J Emerg Med. 2007;25(2):218-219.
15. Arntfield RT, Hicks CM. Systemic Lupus Erythematosus and the Vasculitides. In: Marx JA, ed. Rosen's Emergency Medicine. 8th ed. Philadelphia, PA: Elsevier Saunders; 2014.

16. Borrell H, Narváez J, Alegree JJ, et al. Shrinking lung syndrome in systemic lupus erythematosus: a case series and review of the literature. Medicine (Baltimore). 2016;95(33):e4626. doi:10.1097/ MD.0000000000004626.

17. Aviña-Zubieta JA, Vostretsova K, De Vera MA, et al. The risk of pulmonary embolism and deep venous thrombosis in systemic lupus erythematosus: a general population-based study. Semin Arthritis Rheum. 2015;45(2):195-201. doi:10.1016/j.semarthrit.2015.05.008.

18. Martínez-Martínez MU, Abud-Mendoza C. Predictors of mortality in diffuse alveolar haemorrhage associated with systemic lupus erythematosus. Lupus. 2011;20(6):568-574. doi:10.1177/0961203310392430.

19. Rosenbaum E, Krebs E, Cohen M, Tiliakos A, Derk CT. The spectrum of clinical manifestations, outcome and treatment of pericardial tamponade in patients with systemic lupus erythematosus: a retrospective study and literature review. Lupus. 2009;18(7):608-612. doi:10.1177/0961203308100659.

20. Miner JJ, Kim AH. Cardiac manifestations of systemic lupus erythematosus. Rheum Dis Clin North Am. 2014;40(1):51-60. doi:10.1016/j.rdc.2013.10.003.

21. Morel N, Bonjour M, Le Guern V, et al. Colchicine: a simple and effective treatment for pericarditis in systemic lupus erythematosus? A report of 10 cases. Lupus. 2015;24(14):1479-1485. doi:10.1177/0961203315593169.

22. Roldan CA, Shively BK, Crawford MH. An echocardiographic study of valvular heart disease associated with systemic lupus erythematosus. $N$ Engl J Med. 1996;335(19):1424-1430.

23. Dufrost V, Risse J, et al. Direct oral anticoagulants use in antiphospholipid syndrome: are these drugs an effective and safe alternative to warfarin? A systematic review of the literature. Curr Rheumatol Rep. 2016;18(12):74. doi:10.1007/s11926-016-0623-7.

24. Cervera R, Rodríguez-Pintó I; G Espinosa on behalf of the Task Force on Catastrophic Antiphospholipid Syndrome. Catastrophic antiphospholipid syndrome: task force report summary. Lupus. 2014;23(12):12831285. doi:10.1177/0961203314540764.

25. Sultan SM, Ioannou Y, Isenberg DA. A review of gastrointestinal manifestations of systemic lupus erythematosus. Rheumatology (Oxford). 1999;38(10): 917-932.

26. Xu N, Zhao J, Liu J, et al. Clinical analysis of 61 systemic lupus erythematosus patients with intestinal pseudo-obstruction and/or ureterohydronephrosis: a retrospective observational study. Medicine (Baltimore). 2015;94(4):e419.

27. Vergara-Fernandez O, Zeron-Medina J, MendezProbst C, et al. Acute abdominal pain in patients with systemic lupus erythematosus. J Gastrointest Surg. 2009;13(7):1351-1357. doi:10.1007/s11605-0090897-4.

28. Küçükşahin O, Düzgün N, Okoh AK, Kulahçioglu E. Response to rituximab in a case of lupus associated digital ischemia. Case Rep Rheumatol. 2014;2014:763608. doi:10.1155/2014/763608.

29. Uva L, Miguel D, Pinheiro C, Freitas JP, Gomes MM, Filipe P. Cutaneous manifestations of systemic lupus erythematosus. Autoimmune Dis. 2012;2012:834291. doi:10.1155/2012/834291. 